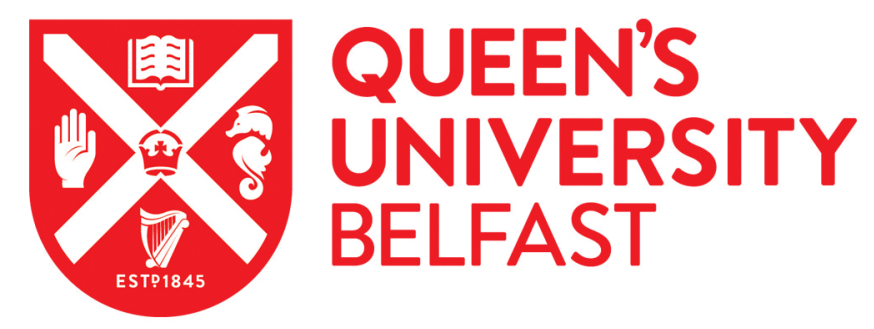

\title{
Electrical and rheological percolation of PMMAMWCNT nanocomposites as a function of CNT geometry and functionality
}

McClory, C., McNally, T., Baxendale, M., Potschke, P., Blau, W., \& Ruether, M. (2010). Electrical and rheological percolation of PMMA/MWCNT nanocomposites as a function of CNT geometry and functionality. European Polymer Journal, 46(5), 854-868. https://doi.org/10.1016/j.eurpolymj.2010.02.009

Published in:

European Polymer Journal

Queen's University Belfast - Research Portal:

Link to publication record in Queen's University Belfast Research Portal

\section{General rights}

Copyright for the publications made accessible via the Queen's University Belfast Research Portal is retained by the author(s) and / or other copyright owners and it is a condition of accessing these publications that users recognise and abide by the legal requirements associated with these rights.

Take down policy

The Research Portal is Queen's institutional repository that provides access to Queen's research output. Every effort has been made to ensure that content in the Research Portal does not infringe any person's rights, or applicable UK laws. If you discover content in the Research Portal that you believe breaches copyright or violates any law, please contact openaccess@qub.ac.uk. 
Provided for non-commercial research and education use. Not for reproduction, distribution or commercial use.

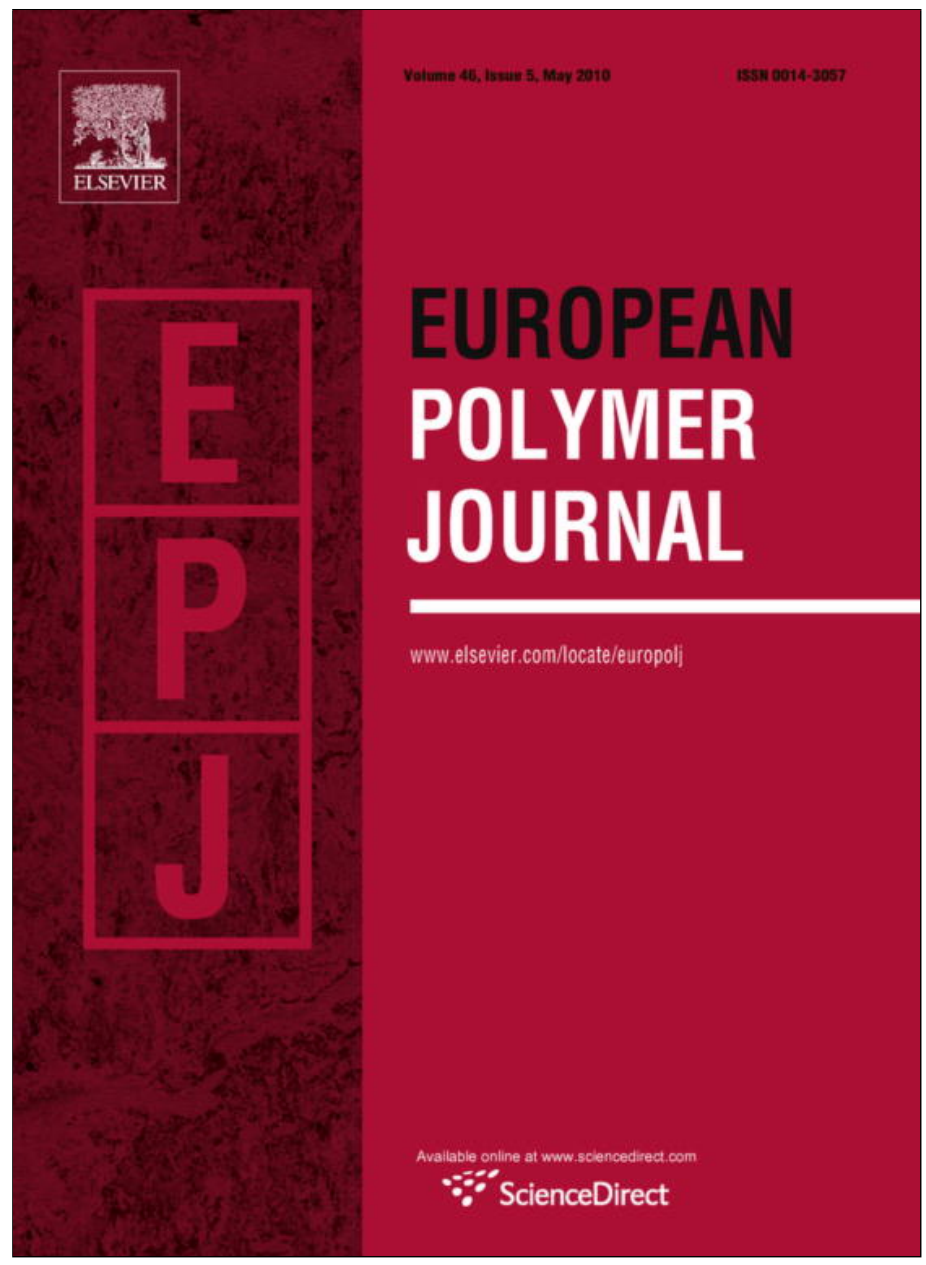

This article appeared in a journal published by Elsevier. The attached copy is furnished to the author for internal non-commercial research and education use, including for instruction at the authors institution and sharing with colleagues.

Other uses, including reproduction and distribution, or selling or licensing copies, or posting to personal, institutional or third party websites are prohibited.

In most cases authors are permitted to post their version of the article (e.g. in Word or Tex form) to their personal website or institutional repository. Authors requiring further information regarding Elsevier's archiving and manuscript policies are encouraged to visit:

http://www.elsevier.com/copyright 
Macromolecular Nanotechnology

\title{
Electrical and rheological percolation of PMMA/MWCNT nanocomposites as a function of CNT geometry and functionality
}

\author{
Caroline McClory $^{\mathrm{a}}$, Tony McNally ${ }^{\mathrm{a}, *}$, Mark Baxendale ${ }^{\mathrm{b}}$, Petra Pötschke ${ }^{\mathrm{c}}$, Werner Blau ${ }^{\mathrm{d}}$, \\ Manuel Ruether ${ }^{\mathrm{d}}$ \\ a School of Mechanical \& Aerospace Engineering, Queen's University Belfast, Belfast BT9 5AH, UK \\ ${ }^{\mathrm{b}}$ Department of Physics, Queen Mary University of London, London E1 4NS, UK \\ ${ }^{c}$ Leibniz Institute of Polymer Research Dresden, Hohe Straße 6, Dresden D-01069, Germany \\ ${ }^{\mathrm{d}}$ Department of Physics, Trinity College Dublin, Dublin 4, Ireland
}

\section{A R T I C L E I N F O}

\section{Article history:}

Received 18 September 2009

Received in revised form 8 February 2010

Accepted 14 February 2010

Available online 18 February 2010

\section{Keywords:}

PMMA

Multi-walled carbon nanotube

Nanocomposite

Melt mixing

Electrical percolation

Rheological percolation

\begin{abstract}
A B S T R A C T
Composites of poly(methyl methacrylate) (PMMA) with multi-walled carbon nanotubes (MWCNT) of varying aspect ratio and carboxylic acid functionality were prepared using melt mixing. The extent of dispersion and distribution of the MWCNTs in the PMMA matrix was investigated using a combination of high-resolution transmission electron microscopy (HRTEM), wide-angle X-ray diffraction (XRD) and Raman spectroscopy. The electrical resistivity and oscillatory shear rheological properties of the composites were measured as a function of MWCNT geometry, functionality, and concentration. The fundamental ballistic conductance of the pristine free-standing MWCNTs was investigated using a mechanically controlled break-junction method. The electrical conductivity of PMMA was enhanced by up to 11 orders of magnitude for MWCNT concentrations below $0.5 \mathrm{wt}$.\%. MWCNTs having higher aspect ratio, above 500, or functionalized with carboxylic acid groups readily formed rheological percolated networks with thresholds, determined from a power law relationship, of 1.52 and 2.06 wt.\%, respectively. The onset of pseudo-solid-like behaviour and network formation is observed as $G^{\prime}, \eta^{*}$, and $\tan \delta^{-1}$ are independent of frequency as MWCNT loading increased. Sufficiently long and/or functionalized tubes are required to physically bridge or provide interfacial interactions with PMMA to alter polymer chain dynamics. Carboxylic acid functionalization disrupts the crystalline order of MWCNTs due to a loss of $\pi$-conjugation and electron de-localisation of $\mathrm{sp}^{2} \mathrm{C}-\mathrm{C}$ bonds resulting in non-ballistic electron transport in these tubes, irrespective of how highly dispersed they are in the PMMA matrix.
\end{abstract}

(c) 2010 Elsevier Ltd. All rights reserved.

\section{Introduction}

Polymeric materials filled with electrically conductive nanoparticles are currently of great interest for use in an extensive range of electronic, aerospace, and military applications, including high strength fibres, in molecular wires, flexible electronics, electromagnetic induction (EMI) shielding, electrostatic dissipation, and as biomolecule sensors. The demand for cost effective production of

\footnotetext{
* Corresponding author. Fax: +44 2890661729.

E-mail address: t.mcnally@qub.ac.uk (T. McNally).
}

these materials has led to an intensive research effort into the melt-processing of polymers filled with conductive fibres and particles. Studies on melt-processed polymer carbon nanotube (CNT) composites are much less common than those produced by in situ polymerization or solution cast methods. A number of thermoplastics have been used in the melt-production of novel polymer/CNT composite materials with improved electrical properties, such as poly(methyl methacrylate) (PMMA) [1], poly(styrene) (PS) [2], medium density poly(ethylene) (MDPE) [3], poly(carbonate) (PC) [4] and others [5,6].

A limited number of previous studies have described the melt mixing of PMMA with CNTs. Jin et al. described 
how up to 26 wt.\% arc-discharge produced MWCNTs were dispersed in PMMA using a 'Laboratory Mixing Moulder' at $200{ }^{\circ} \mathrm{C}$ [1]. TEM studies revealed the nanotubes to be undamaged by the mixing process and relatively well dispersed with no large agglomerations present. The addition of 26 wt.\% CNTs to PMMA delayed the onset of thermal degradation by $30 \mathrm{~K}$ and an improvement of $2700 \%$ in storage modulus at $120^{\circ} \mathrm{C}$ was reported. Gorga and Cohen dry blended PMMA with single walled carbon nanotubes (SWCNTs) and MWCNTs before extruding the mixtures at $230^{\circ} \mathrm{C}$ through a cylindrical die and reported good dispersion at low nanotube loadings [7]. Successful alignment of nanotubes was also observed by melt drawing the extrudate, resulting in an increase in tensile toughness of $170 \%$ with the addition of $1 \mathrm{wt} . \%$ orientated MWCNTs with respect to the original polymer. Haggenmueller et al. used a combination of solvent casting and melt mixing to prepare PMMA/SWCNT films and fibres for electrical and mechanical testing [8]. The electrical conductivity measured in the direction of flow of molten material during compression moulding was found to increase from 0.118 to $11.5 \mathrm{~S} / \mathrm{m}$, as the nanotube loading increased from 1.3 to $6.6 \mathrm{wt} . \%$, respectively. However, measurements made normal to the direction of flow gave significantly lower values. The elastic modulus and yield strength of the highly oriented PMMA/SWCNT melt spun fibres increased with nanotube loading and draw ratio.

Functionalization of nanotube surfaces has been used as a method of improving compatibility of MWCNTs with polymers. Zhou et al. directly modified the surface of MWCNTs with PMMA via emulsion polymerization initiated by $2,2^{\prime}$-azoisobutyronitrile (AIBN) in an aqueous solution of sodium laurel sulphate (SLS), then made a range of composites by further melt mixing with PMMA[9]. The rheological percolation threshold was reduced from 2.5 to $1.5 \mathrm{wt}$ \% by using PMMA-modified MWCNTs. Jin et al. used poly(vinylidene fluoride) PVDF as a coupling agent for MWCNTs and PMMA [10], which yielded a doubling of the storage modulus at $50{ }^{\circ} \mathrm{C}$ by incorporating $0.5 \mathrm{wt} . \%$ PVDF. Park et al. used acid-treated, catalytic chemical vapour deposition (CVD) produced MWCNTs and AIBN as a radical initiator to synthesize PMMA/CNT composites by in situ polymerization [11]. The authors reported the evolution of a new peak in the Raman spectrum of the composite, evident at $1650 \mathrm{~cm}^{-1}$ and originating from the formation of a $\mathrm{C}-\mathrm{C}$ bond between the MWCNTs and PMMA, as a consequence of the polymerization reaction. A four point probe method was used to measure the electrical properties of the composites and the conductivity increased significantly with the addition of MWCNTs from $1 \times 10^{-13} \mathrm{~S} / \mathrm{cm}$ for insulating PMMA, to $3.2 \times 10^{-4}, 2.2 \times$ $10^{-2}$, and $1.7 \times 10^{-1} \mathrm{~S} / \mathrm{cm}$ for 1,5 and $10 \mathrm{wt} . \%$ nanotube loadings, respectively. The DC conductivity of PMMA/ MWCNT samples prepared by solution mixing were reported by Kim et al., the incorporation of both Fe- and Co-CVD produced MWCNTs was investigated [12]. Films were made by the ultrasonication of MWCNTs, PMMA, and toluene over a period of $24 \mathrm{~h}$ and subsequent solvent extraction. The distribution of the nanotubes was described as 'homogeneous' although no evidence of this was provided in the paper. However, the electrical conductivity results obtained, again using a four point probe method, suggested a percolated network of nanotubes had formed throughout the polymer film with a percolation threshold estimated at $\sim 0.003 \mathrm{wt} . \%$. Log-log plots of DC conductivity versus mass fraction of Fe-MWCNTs revealed the conductivity $(\sigma)$ of PMMA increased by $\sim 10^{10} \mathrm{~S} / \mathrm{cm}$ on addition of $0.3 \mathrm{wt} . \%$ CNTs and the critical exponent of the percolated network was $\sim 2.15$. In other work by Benoit et al. [13] a percolation threshold of $0.33 \mathrm{wt} . \%$ was reported for PMMA/SWCNT solution cast composites in tests performed on $10 \mu \mathrm{m}$ thick samples. Du et al. reported the alignment of SWCNTs in a PMMA composite reduced the electrical conductivity of a $2 \mathrm{wt} . \%$ composite from $10^{-4} \mathrm{~S} / \mathrm{cm}$ (unaligned) to $10^{-10} \mathrm{~S} / \mathrm{cm}$ (aligned) [14]. The electrical conductivity was decreased due to the reduction in the number of electrical contacts as a consequence of nanotube orientation.

The CNT loadings required to achieve a percolated network of conducting particles by melt mixing is significantly higher than previously reported for other polymer/ MWCNTs composites produced by in situ polymerisation and solution processing [15-17]. In these papers, 15-17, the authors, using an epoxy as the host matrix, reported an improved electrical conductivity of about 9 orders of magnitude compared to the neat resin, for percolation thresholds occurring well below 0.05 wt.\% CNTs. In particular, Sandler et al. using aligned multi-walled carbon nanotubes in an epoxy material achieved an ultra-low percolation threshold of 0.0025 wt.\% [16]. Mc Nally et al. [3] have proposed that the higher electrical percolation thresholds observed for melt-mixed polymers/CNT composites compared to those prepared using solution and in situ polymerisation processes is a consequence of the transfer of electrons required for conduction throughout the bulk sample being hindered by a layer of insulating polymer (in the order of nanometres thick), observed from HRTEM studies, that coats the surface of nanotubes. This is of signifcant importance for semi-crystalline polymers as CNTs can act as nucleating sites for crystallisation. Furthermore, the melt viscosity of the polymer will also play a role with regard the formation of a polymer-rich coating on CNTs during melt mixing. A critical minimum distance is required between next nearest neighbour nanotubes to facilitate electron hopping or tunnelling. The maximum tunnelling distance, from Monte Carlo simulations, was recently reported to be $1.8 \mathrm{~nm}$, although this will be dependent on the inherent electrical conductivity of the polymer and nanotube chirality [18]. Alignment of nanotubes from the extrusion process is also considered to be a contributing factor as to whether an electrical and/or rheological percolation is obtained, although conversely, as mentioned above, Sandler et al. reported using aligned MWCNTs a very low electrical percolation threshold (0.0025 wt.\%) in an epoxy matrix[16].

In this paper we report the preparation of melt-processed PMMA/MWCNT composites as a function of nanotube geometry and functionality. The composite morphology and the extent of the dispersion of the MWCNTs in the PMMA matrix were correlated with electrical and rheological property measurements to elucidate the formation of a percolated network. The electron transport of the pristine MWCNTs is determined from ballistic conductance measurements at room temperature. 


\section{Experimental}

\subsection{Materials and composite preparation}

The PMMA used in the study was Diakon ${ }^{\mathrm{TM}}$ CMG302 supplied by Distrupol Ireland and produced by Lucite International Inc. with $\mathrm{MFI}=4.4 \mathrm{~g} / 10 \mathrm{~min}\left(200^{\circ} \mathrm{C} / 5.0 \mathrm{~kg}\right.$ ) (ISO 1133), average molecular weight $\left(M_{\mathrm{w}}\right) 85,000 \mathrm{~g} / \mathrm{mol}$. (GPC) and $\rho=1.18 \mathrm{~g} / \mathrm{cm}^{3}$ (ISO 1183). It is a general purpose moulding and extrusion grade PMMA with high heat resistance used mainly in the production of optical parts, display items, and for tube and profile extrusion. It is relatively hygroscopic, absorbing $0.3 \%$ of its mass in water and as a consequence the PMMA and all MWCNT materials used were dried for at least $24 \mathrm{~h}$ at $80^{\circ} \mathrm{C}$ prior to melt-processing. In the first instance and in order to investigate whether the initial polymer particle size affected the properties of the PMMA/MWCNT composites prepared, it was necessary to reduce the size of the particles from pellet form $(\mathrm{mm})$ to fine powder $(\mu \mathrm{m})$ prior to melt mixing. A Wedco SE-12-TC grinding machine was used to reduce the PMMA pellet size to a fine powder having an average particle size of $250 \mu \mathrm{m}$, rendering it more easily blended with MWCNT powder prior to melt mixing. The synthesis methods used to prepare the MWCNTs used in this study, their properties and dimensions are listed in Table 1. A Collins ZK25 (30:1 L/D ratio) twin-screw extruder $(100 \mathrm{rpm})$ was used to prepare all PMMA/MWCNT composites with nanotube loadings from 0.01 to $10 \mathrm{wt} . \%$. An intermeshing, co-rotating, twin screw configuration was employed for all composites, generally accepted as an effective method of achieving high levels of dispersion and distribution of nanoparticles in molten polymers [19]. On exiting the capillary die, the extrudate entered a bath of cooled water and was drawn off at a constant rate and pelletised. Bulk composite samples were fabricated into sheet form using a Rondol manual compression moulder and test specimens cut from this sheet.

\subsection{Composite characterization}

The nanotubes, before and after melt mixing, the extent of dispersion and their distribution in the PMMA matrix were assessed using a combination of high-resolution transmission electron microscopy (HRTEM) and wide-angle X-ray diffraction (WAXD). Specimens for HRTEM examination ( $\sim 90 \mathrm{~nm}$ thick) were microtomed using a diamond knife from moulded samples using an Ultracut-E Reichert Jung ultramictrotome. HRTEM characterisation was carried out using an FEI Tecnai F20 field emission high-resolution transmission electron microscope using an accelerating voltage of $200 \mathrm{kV}$. The destruction of the long range lamellar and concentric arrangement of the MWCNTs post melt mixing with PMMA was studied using WAXD operating a PANalytical X'Pert Pro Siemens D5000 diffractometer using $\mathrm{Cu}-\mathrm{K}_{\alpha}$ radiation $(\lambda=1.5406 \AA)$ and a scanning rate of $1 \mathrm{~min}^{-1}$ in the range $1-40^{\circ} 2 \theta$. Raman spectra of the unfilled PMMA, MWCNTs, and all nanocomposites were collected using an Avalon Instruments Raman Station R1 fitted with an 8200 Detector Element Echelle CCD detector. The system was capable of collecting spectra over a Raman shift spectral range of approximately $250-3500 \mathrm{~cm}^{-1}$. Volume resistivity measurements were made on compression moulded films with thicknesses of 100 and $250 \mu \mathrm{m}$. To avoid temperature effects and the interference of electrical noise, high resistivity testing was performed in accordance with ASTM-D257 and experiments conducted using a high accuracy Keithley 6517A electrometer and a shielded test enclosure (Keithley 8009 resistivity test fixture). For samples of low resistivity, two-point probe measurements were used on the same principle as the volume resistivity test set-up using the Keithley 6517A electrometer but in combination with two-point probes and the assistance of platinum or silver contacts, in order to minimise contact resistance effects. All measurements were made in triplicate.

\subsection{Ballistic conductance of pristine MWCNTs}

Electrical conductance of the pristine, free-standing nanotubes used in this study was probed by a mechanically controlled break-junction method, the detail of the experimental configuration and data interpretation has been reported previously [20]. The bias voltage for these measurements was selected to be below the threshold for sub-band injection in MWCNTs previously identified by Poncharal et al. [21]. The method compiles conductance histograms for ballistic conduction in networks for carbon

Table 1

Sample codes and characteristics of MWCNT materials.

\begin{tabular}{|c|c|c|c|c|c|c|c|c|}
\hline Supplier (grade) & CODE & $\begin{array}{l}\text { Synthesis } \\
\text { method }\end{array}$ & $\begin{array}{l}\text { CNT content } \\
(\%)\end{array}$ & $\begin{array}{l}\text { Carbon } \\
\text { purity (\%) }\end{array}$ & $\begin{array}{l}\mathrm{COOH} \text { funct. } \\
(\%)\end{array}$ & $\begin{array}{l}\text { Diameter } \\
(\mathrm{nm})\end{array}$ & $\begin{array}{l}\text { Length } \\
(\mu \mathrm{m})\end{array}$ & $\begin{array}{l}\text { Aspect } \\
\text { ratio }\end{array}$ \\
\hline $\mathrm{TCD}(\mathrm{n} / \mathrm{a})$ & CNT-A & $A D^{a}$ & $15^{d}$ & - & - & $2-10$ & $<1$ & $\begin{array}{l}100-500 \\
300^{\mathrm{e}}\end{array}$ \\
\hline $\begin{array}{r}\text { MER Corp } \\
\text { MRGC }\end{array}$ & CNT-B & $\mathrm{AD}^{\mathrm{a}}$ & $30-40^{c}$ & - & - & $6-20$ & $1-5$ & $\begin{array}{l}50-833 \\
442^{\mathrm{e}}\end{array}$ \\
\hline $\begin{array}{l}\text { Sun Nanotech }^{\circledR} \\
\text { MWCNT-L-P }\end{array}$ & CNT-C & $\mathrm{CCVD}^{\mathrm{b}}$ & $>90$ & $>95$ & - & $10-30$ & $1-10$ & $\begin{array}{l}33-1000 \\
517^{\mathrm{e}}\end{array}$ \\
\hline $\begin{array}{l}\text { Nanocyl }^{\circledR} \\
\quad \text { Nanocyl }^{\circledR}-3101\end{array}$ & CNT-D & $\mathrm{CCVD}^{\mathrm{b}}$ & $>95$ & $>95$ & $<4$ & $\sim 10$ & $<1$ & 100 \\
\hline
\end{tabular}

\footnotetext{
a Arc-discharge.

b Catalytic chemical vapour deposition.

c Impurities bucky-onions and graphite particles.

d Amorphous carbonaceous material.

e Average aspect ratio.
} 
nanotubes from a statistically large data set. Conductance is expressed in terms of the conductance quantum $G_{0}=2 e^{2} / h\left(G_{0}{ }^{-1}=12,900 \Omega\right)$ and data is interpreted in terms of series and parallel combinations of the fundamental conductance and transmission coefficient per nanotube [20]. The method follows from the landmark paper of Frank et al. and subsequent publications, which found electron transport in MWCNT bundles to be ballistic at room temperature [22-27]. The ballistic conductance regime is governed by the Landauer-Büttiker equation [28]:

$G=G_{0} \sum_{\alpha \beta} T_{\alpha \beta}$

where $T_{\alpha \beta}$ is the transmission probability of an electron going from channel $\alpha$ to channel $\beta$ with $\alpha$ and $\beta$ representing the excited bands involved in the transport [28-30]. Theory predicts two conductance channels for an ideal metallic SWCNT [31]. For an ideal SWCNT with ideal contacts the transmission coefficient for both conductance channels will equal unity, hence, for ideal SWCNTs Eq. 1 becomes $G=2 G_{0}$. For a non-ideal contacted SWCNT the transmission coefficients can be depressed below unity by backscattering in the nanotube and imperfect contacts. Similarly, point defects and surface contaminants can depress conductance by $\sim \Delta G_{0}=1$ for a $1.4 \mathrm{~nm}$ diameter SWCNT [32]. Alternatively the origin of non-integer- $G_{0}$ quantisation can be explained in terms of a simple series ballistic wire model in which the total conductance can be expressed as:

$G=G_{0}\left(\sum\left(n_{j}\right)^{-1}\right)^{-1}$

where the summation is over the number of wires in series and $n_{j}$ is the number of channels in wire $j$, assuming unity transmission probabilities for all wires [27].

Oscillatory rheological properties of the samples were determined using an ARES-rheometer (Rheometrics Scientific). The measurements were performed in dynamic mode under a liquid nitrogen atmosphere using $25 \mathrm{~mm}$ parallel plate geometry and a constant gap width of approximately $1.5 \mathrm{~mm}$. Compression moulded $25 \mathrm{~mm}$ polymer discs were used and the tests performed at $220^{\circ} \mathrm{C}$. Rheological properties were measured as a function of angular frequency which was varied from 0.1 to $100 \mathrm{rad} / \mathrm{s}$ and the strain amplitude was chosen to be within the viscoelastic range of the composites.

\section{Results and discussion}

In the first instance, we attempted to ascertain the significance of the particle size (pellet versus powder) of the PMMA used during mixing on the extent of dispersion of MWCNTs in the matrix, which in turn governs percolation threshold. Fig. 1a and b show HRTEM images of CNT-C (8.5 wt.\%) melt-mixed with PMMA in pellet and powder form, respectively, prepared using identical processing conditions. It was obvious that a better state of CNT dispersion and distribution was achieved when the PMMA had a smaller initial particle size ( $\approx 250 \mu \mathrm{m}$ compared to $1-2 \mathrm{~mm}$ ), therefore the PMMA used in this study was ground to a powder prior to melt mixing with all MWCNTs. The PMMA was characterised using Fourier transmission infra-red spectrometry (FTIR) before and after grinding and confirmed the polymer had not degraded during the relatively high-friction grinding process. In Fig. 1a, large areas of agglomerated CNT-C were observed with isolated CNTs dispersed in the PMMA matrix close to these agglomerations. Melt-processing was not effective in fully disentangling and distributing CNT-C bundles and tubes when starting from PMMA in pellet form. The degree of nanotube dispersion can be sub-divided into three categories; (1) regions of well dispersed nanotube material, (2) nanotube agglomerations and (3) a PMMA-rich phase. The HRTEM image in Fig. 1b clearly shows how the reduced PMMA particle size assisted nanotube dispersion, as the CNTs appear disentangled and well dispersed throughout the polymer matrix. Exhaustive microscopic examination of the composite across the length scales did show some regions void of nanotubes or filled with smaller agglomerations sub- $1 \mu \mathrm{m}$ in diameter, although in all cases the CNTs were much better dispersed and distributed than for the composite prepared with PMMA in pellet form. Representative HRTEM images of the composites prepared with arc discharge (AD) tubes (CNT-A and CNT-B) and the carboxylic acid functionalised MWCNTs (CNT-D) are shown in Fig. 1c-f. The shorter more rigid, smaller aspect ratio $\mathrm{AD}$ CNT-A and CNT-B tubes are clearly evident in Fig. 1c and 1d, and CNT-A is well dispersed in the PMMA matrix. The slightly higher aspect ratio CNT-B tubes are less well dispersed, but for both CNT-A and CNT-B significant levels of impurities are also evident (dark regions). The CNT-B tubes were also coated with a PMMA-rich layer as the diameters of the structures shown are larger than that of CNT-B alone. The functionalised tubes, CNT-D, were also highly dispersed in the PMMA matrix, see Fig. 1e and f. Moreover, these tubes appear to align to an extent in the extrusion (shear) direction. Presumably, this behaviour is governed by both the electrostatic repulsion between carboxyl groups on the CNT tips and the increased interfacial interactions between CNT-D and polar PMMA chains. Similarly, the carboxylic acid functionalization of MWCNTs in a study by Gojny et al. improved the dispersion of MWCNTs in an epoxy matrix due to the repulsive forces [33]. Amide functionalisation of MWCNTs has also been shown to aid dispersion of MWCNTs in PMMA via solution mixing, however, the electrical resistance of the composite material increased with increased MWCNT loading [34].

The effectiveness of the mixing process in shearing and disentangling the CNT bundles was examined by WAXD, see Fig. 2. The four MWCNT samples used in this study displayed a sharp diffraction peak $\left(\begin{array}{lll}0 & 02\end{array}\right)$ at $2 \theta=26^{\circ}$ corresponding to the ordered arrangement of the concentric cylinders of graphitic carbon [35]. This peak was much sharper for the $\mathrm{AD}$ produced tubes as $\mathrm{AD}$ tubes have less defect sites and a more perfect honeycomb lattice structure compared to CVD produced tubes. This reflection peak was absent for the composites with a low loading of CNT-A and CNT-B, typically below 1 wt.\%, but was clearly observed for loadings above $1 \mathrm{wt} . \%$. Furthermore, for PMMA/CNT-A composites the peak shifted to a slightly higher $2 \theta$ value, and for PMMA/CNT-B, the peak split in two, evidence for the effect of shear forces tearing 

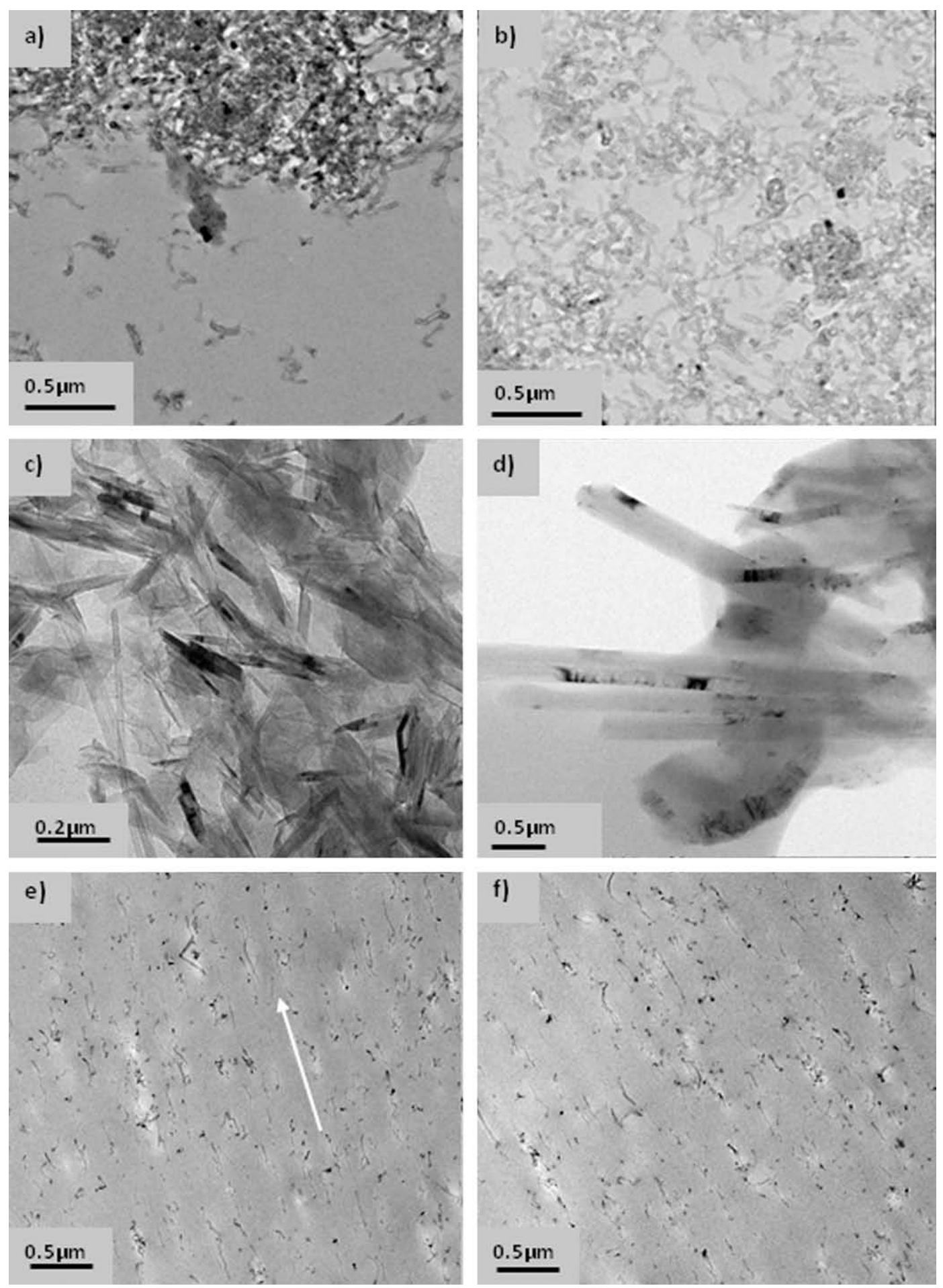

Fig. 1. HRTEM images of 8.5 wt.\% composites of PMMA (a) in pellet form with CNT-C, (b) in powder form with CNT-C, and 5 wt.\% composites of PMMA with (c) CNT-A, (d) CNT-B, (e) and (f) CNT-D.

and distorting the CNTs during mixing in the compounder.

AD produced MWCNTs are by nature needle-like, uniform, crystalline and retain their short-range order even after high-shear melt-processing. The 002 peak became narrower on composite formation as the short-range order of the nanotubes was disturbed on addition to PMMA, due to the tearing and shearing forces encountered during melt mixing. The shear applied during mixing was less effective at disentangling the tubes as the loading of smaller diameter more rigid $\mathrm{AD}$ tubes increased. Furthermore, it appears the melt mixing process did not initiate tube scission, as the integrity of the nanotubes was retained after composite formation, see Fig. 1. CNT-A and CNT-B in their pristine state have a corresponding d-spacing of $3.398 \AA$ which is known to be the interlayer spacing 

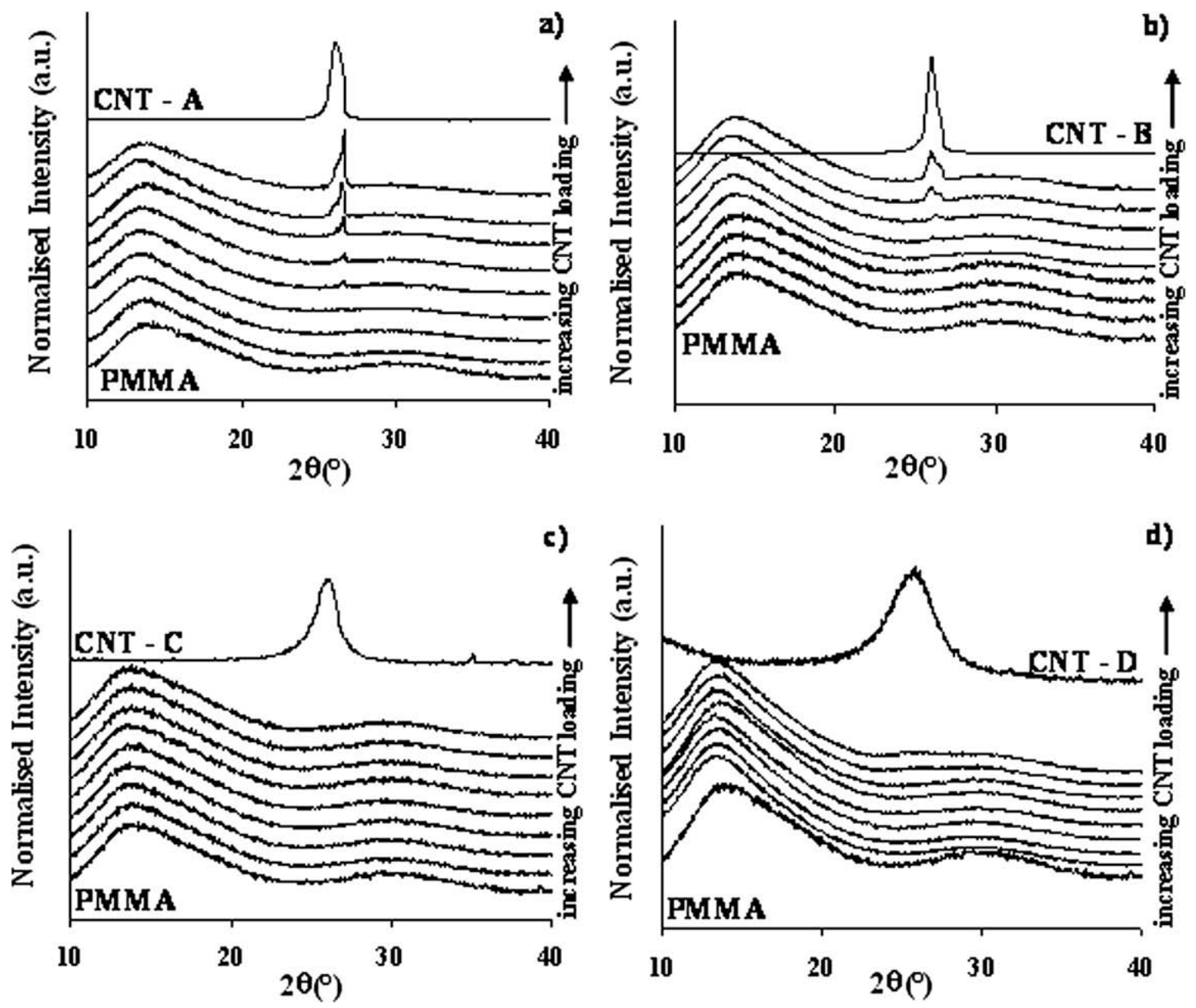

Fig. 2. WAXD patterns of composites of PMMA with (a) CNT-A, (b) CNT-B, (c) CNT-C and (d) CNT-D in the range of 2-40 ${ }^{\circ}$ with increasing MWCNT content (1-10 wt.\%).

between concentric nanotubes within the multi-layered structure. After melt-processing this value decreased to $3.348 \AA$ as the tubes embedded in the polymer matrix were constricted and under the compressive strain of the surrounding matrix. The short-range order of CVD produced MWCNTs was lost on composite formation as the 002 reflection was not observed for the composites containing CVD produced tubes, see Fig. $2 \mathrm{c}$ and $\mathrm{d}$.

The effect of shear forces during mixing on the integrity of the tubes and the extent of interactions between the PMMA matrix and the different MWCNTs was investigated using Raman spectroscopy. MWCNTs, like all graphitic materials exhibit $D$ and $G$ bands in their Raman spectra associated with defect density $(D)$ and the tangential stretching mode $(G)$, respectively. Fig. 3a and b shows the normalised Raman spectra of the composites prepared with PMMA in pellet and powder form with CNT-C, respectively. The D and G bands were observed for both sets of composites, the relatively intense $\mathrm{D}$ band confirming the high levels of defects sites in CVD produced tubes. Interestingly, the intensity of the $\mathrm{D}$ band increased to an approximate constant value independent of CNT-C loading, implying the defect density of these tubes increased as a consequence of the melt mixing process. The defect density can be estimated by rationing the relative intensities of the $\mathrm{D}$ and $\mathrm{G}$ bands and generating a plot of $I_{\mathrm{D}} / I_{\mathrm{G}}$ against wt.\% MWCNT, see Fig. 3c. For pristine CNT-C $I_{\mathrm{D}} / I_{\mathrm{G}}=0.53$, as $I_{\mathrm{D}}$ increased $I_{\mathrm{D}} / I_{\mathrm{G}}$ increased to between 1.4 and 1.5 irrespective of CNT-C loading. The G band, for the PMMA (powder)/CNT-C composites, up-shifted by about $20 \mathrm{~cm}^{-1}$. This up-shift is a result of the polymer matrix exerting a compressive force on the nanotubes, altering the tangential motion of the tubes, implying the polymer is intercalated between tubes, there are interfacial interactions between PMMA chains and CNT-C, and the tubes are well dispersed in the PMMA matrix, supported by HRTEM studies, see Fig. 1b. The Raman spectra of the composites prepared with CNT-A, CNT-B and CNT-D are shown in Fig. $4 a-c$, respectively. The $D$ band for the composites containing both $\mathrm{AD}$ produced tubes, CNT-A and CNT-B, was less intense than that obtained for the CVD produced tubes. Furthermore, there was little shifting of the $G$ band. $I_{\mathrm{D}} / I_{\mathrm{G}}$ for both $\mathrm{AD}$ produced tubes was 0.36 , lower than that determined for the CVD produced CNT-C tubes (0.53). The lower number of defects detected for the $\mathrm{AD}$ produced MWCNTs is in agreement with the increased perfection 
a)

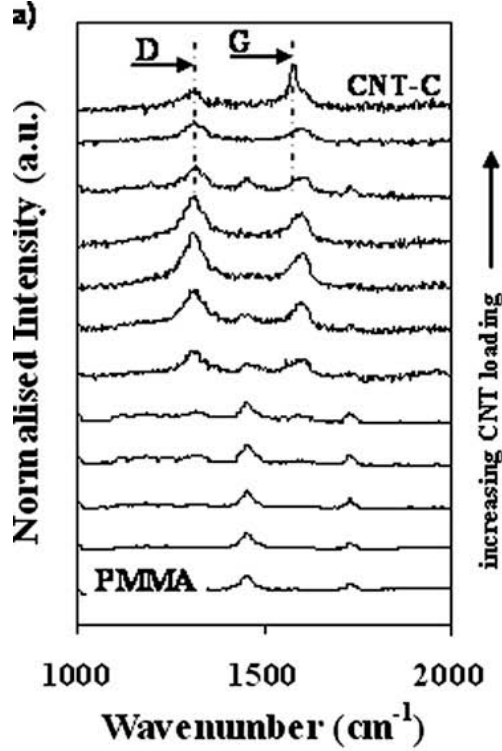

b)

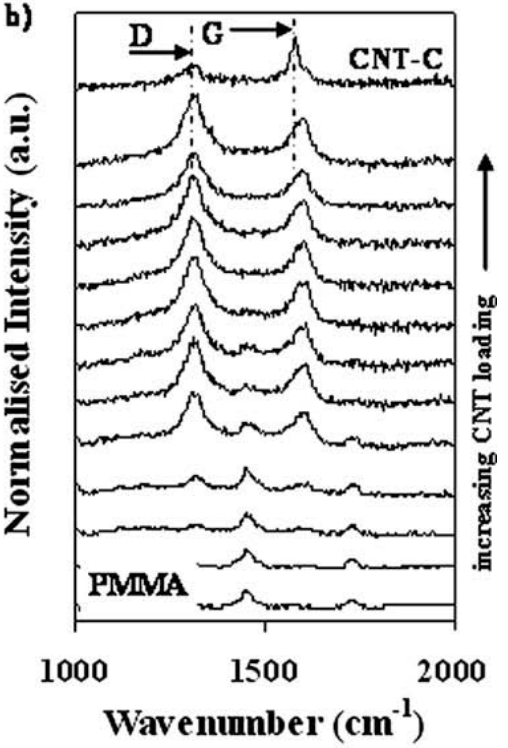

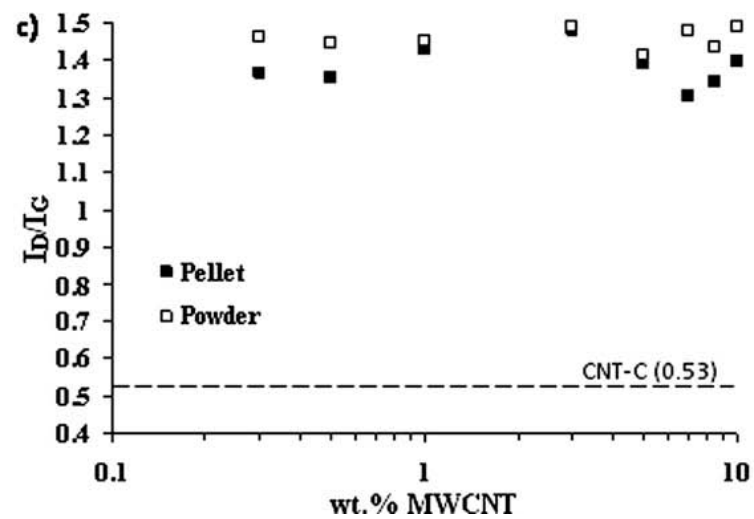

Fig. 3. Raman spectra of composites of CNT-C with (a) PMMA in pellet form (b) PMMA in powder form, in the range of $1000-2000 \mathrm{~cm}^{-1}$, (c) $I_{\mathrm{D}} / I_{\mathrm{G}}$ Raman ratios of for both set of composites in (a) and (b).

of the honeycomb lattice structure reported previously for arc-discharge produced MWCNTs [36]. $I_{\mathrm{D}} / I_{\mathrm{G}}$ of the composites was reduced significantly as nanotube content was increased, an effect more pronounced for the composites containing higher aspect ratio nanotubes. The decrease in $I_{\mathrm{D}} / I_{\mathrm{G}}$ for the PMMA/CNT-A and PMMA/CNT-B composites is due to an increase in the intensity of the tangential mode ( $G$ band) of the MWCNTs within the composites relative to the D-band, as a function of increasing nanotube loading. Valentini et al. for a range of PP/SWCNT composites processed by melt-extrusion proposed that the general increase in G-band intensity was due to a reduction in the quantity of polymer intercalated between the nanotube bundles as CNT loading was increased [37]. However, for isotropically ordered SWCNT shells an increase in G-band intensity is more likely an indication of the breakage of SWCNT shells. The hypothesis proposed by Valentini et al. is valid for composites of de-bundled MWCNTs and polymers.

The extent of formation of an electrical percolated network was studied by measuring the volume resistivity of the PMMA/MWCNT composites as a function of CNT loading, up to $10 \mathrm{wt. \%}$, see Fig. 5. Only PMMA/CNT-A and
PMMA (powder)/CNT-C systems attained an electrical percolated network with thresholds $\left(\rho_{\mathrm{c}}\right)$ at 0.5 and 7.75 wt.\%, respectively, further supporting HRTEM observations that CNT-A and CNT-C were highly dispersed in the PMMA matrix. The volume resistivity decreased by about 11 orders of magnitude from $10^{17}$ to $10^{6} \mathrm{Ohm} \mathrm{cm}$ on addition of either CNT-A or CNT-C. As pristine CNT-A was only $15 \%$ MWCNT pure and assuming only metallic MWCNTs contributed to the electrically conducting network, the actual percolation threshold $\left(\rho_{\mathrm{c}}\right)$ is approximately $0.075 \mathrm{wt} . \%$. Again the intimate mixing of PMMA powder with CNT-C facilitated more readily the formation of a network of the high aspect ratio entangled CVD produced CNT-C. The composites prepared with CNT-B and CNT-D did not form an electrical percolated network. That CNT-D was highly dispersed in the PMMA matrix yet no electrically conducting percolated network was obtained can be explained in terms of how the carboxylic acid functional groups destroy electron hoping and tunnelling. The excellent dispersion achieved did not lead to the formation of a conducting network of nanotubes and hence the functionalised MWCNTs did not alter the resistivity of the neat polymer. The addition of functional groups to a CNT has been known to disturb the 

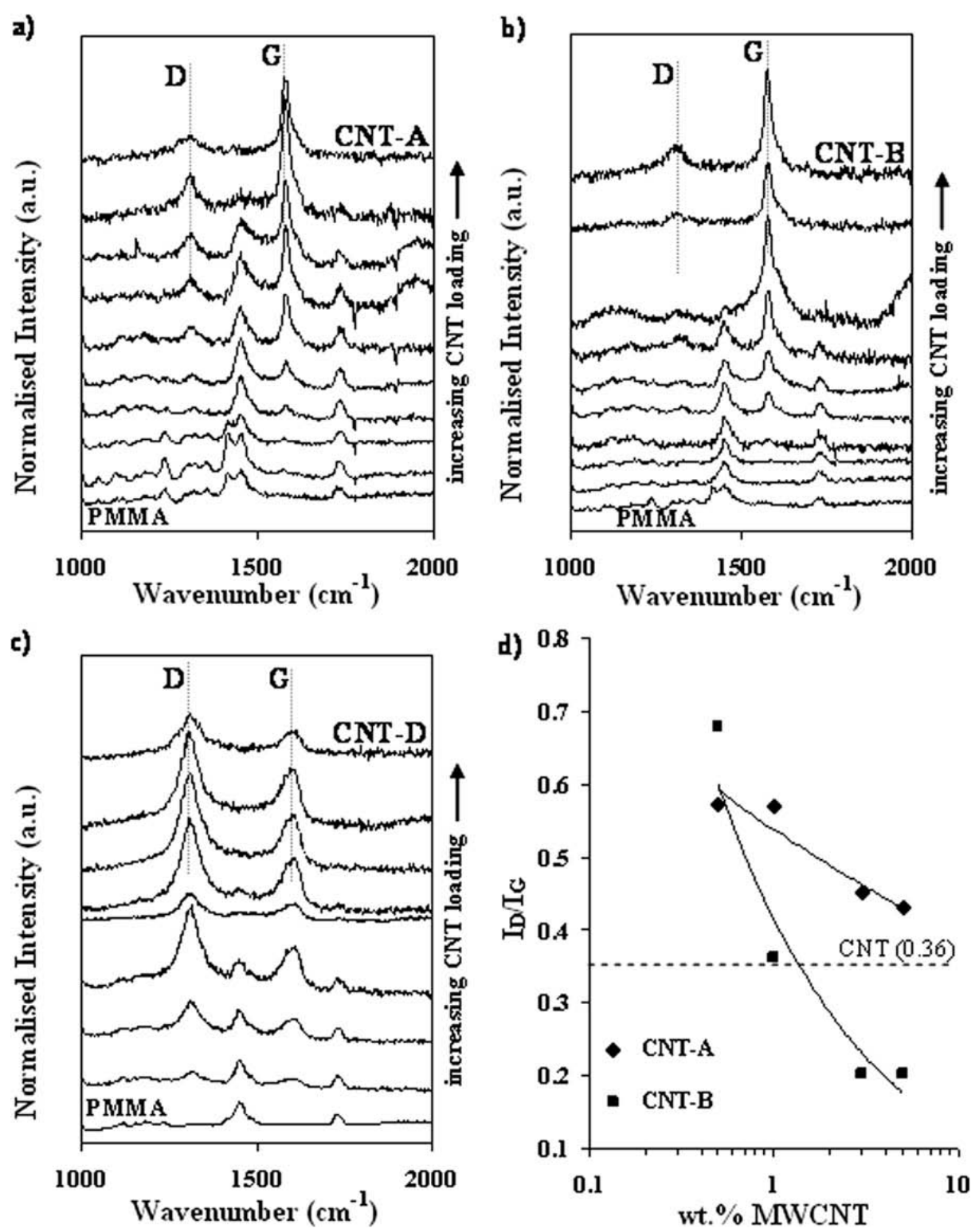

Fig. 4. Raman spectra of composites of PMMA with (a) CNT-A, (b) CNT-B, (c) CNT-D, in the range of $1000-2000 \mathrm{~cm}^{-1}$, (d) $I_{\mathrm{D}} / I_{\mathrm{G}}$ Raman ratios of PMMA/CNT-A and PMMA/CNT-B composites.

graphitic structure of the nanotube by introducing $\mathrm{sp}^{3}$ hybridization to the conjugated system [38]. This change in the fundamental structure of the nanotube disrupted the conjugation and hence induced a distortion of the graphitic layer. In turn, these $\mathrm{sp}^{3}$ bonded sites are viewed as defects in terms of electron transport through a nanotube and result in a reduction in the overall electrical conductivity of the nanotube [38]. Additionally, the promoted interactions between polymer chains and functional groups on the surface of a nanotube may account for the overall reduction in electrical conductivity as a layer of insulating polymer may impede electron flow by increasing the distance required for an electron to travel from tube to tube. As stated previously, recent Monte Carlo studies have provided an estimate of the maximum tunnelling distance is such composites to be about $1.8 \mathrm{~nm}$ [18].
The fundamental ballistic conductance of the pristine free-standing tubes were thus measured, see Fig. 6 . The $x$-axis of the plots is conductance in units of the conductance quantum and the $y$-axis is the quantity $\mathrm{H}$ which is a measure of the frequency of occurrence of the conductance value within the sample [20]. Fig. 6a shows the conductance histogram for CNT-A, (note that $H$ is displayed on a logarithmic scale); the histogram is dominated by a broad feature close to zero conductance with minor peaks at $0.38 \pm 0.03 G_{0}, 0.60 \pm 0.04 G_{0}, 1.04 \pm 0.09 G_{0}, 2.25 \pm 0.20 G_{0}$, $3.32 \pm 0.30 G_{0}$, and $4.44 \pm 0.25 G_{0}$. The main broad feature close to zero conductance is attributed to non-quantised diffusive conduction, with superimposed minor peaks and indicates some contribution from ballistic transport. Although skewed by this broad feature, the minor peaks can be interpreted by the series and parallel ballistic 


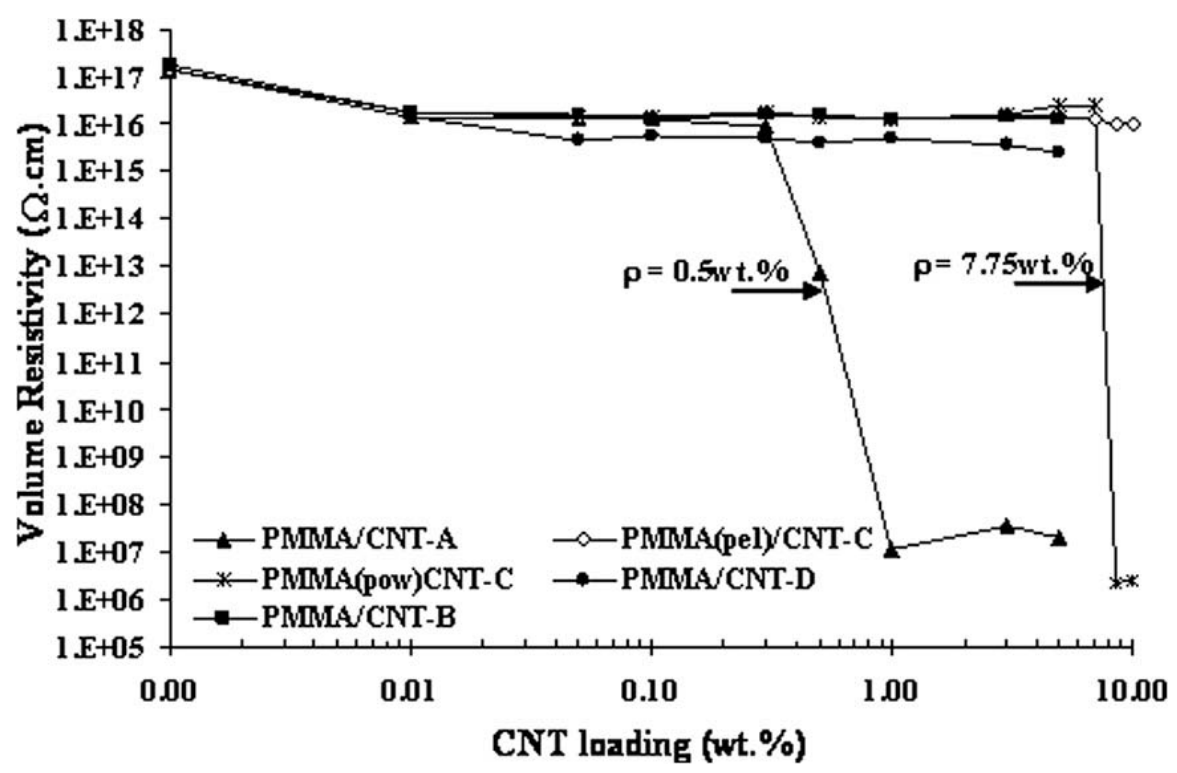

Fig. 5. Variation in volume resistivity as a function of CNT loading, for different PMMA/MWCNT composites, sample thickness $250 \mu$ m.
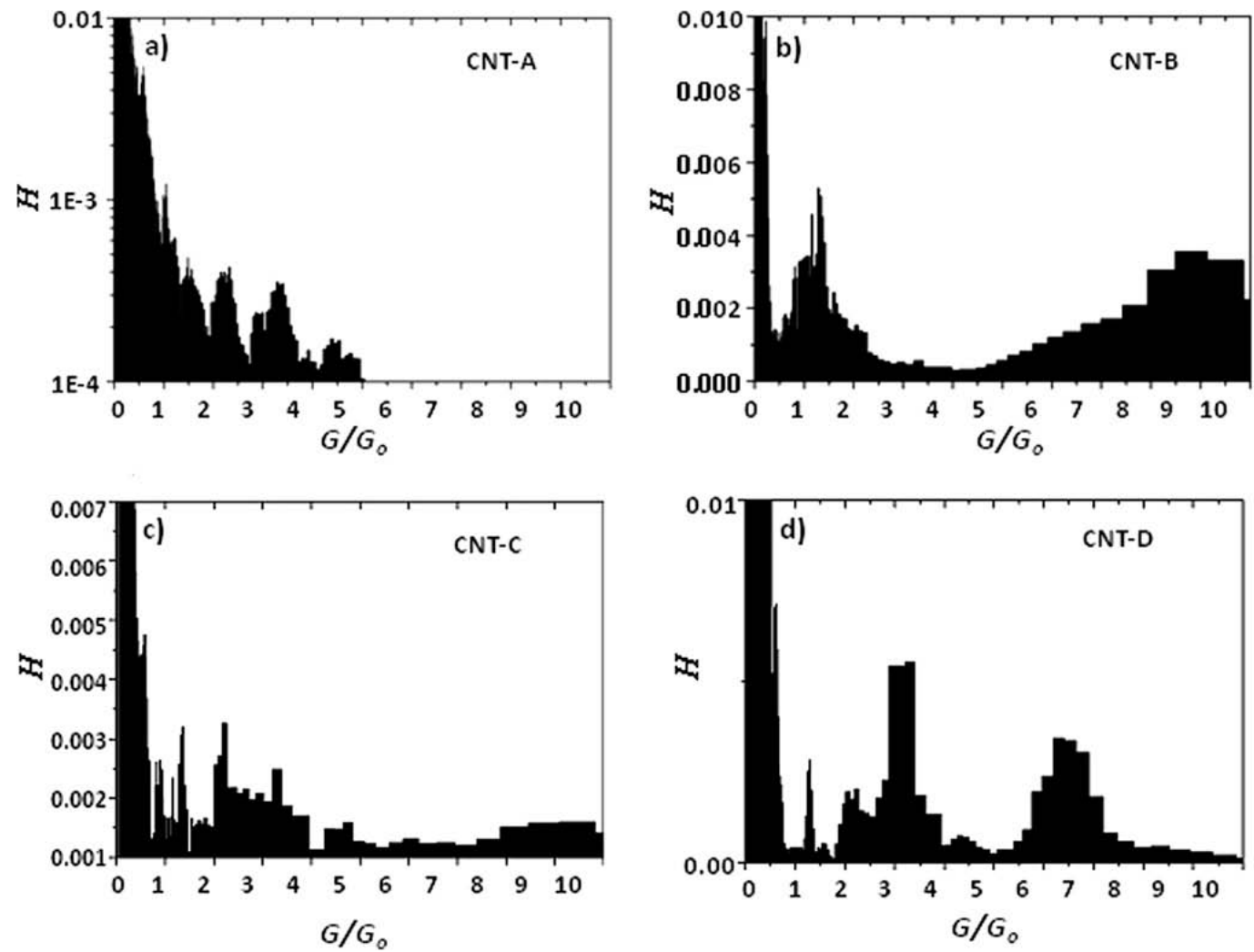

Fig. 6. Ballistic conductance behaviour for composites of PMMA with (a) CNT-A (b) CNT-B (c) CNT-C and (d) CNT-D.

wire model with fundamental conductance values at $1.04 \pm 0.09 G_{0}$ and $2.25 \pm 0.20 G_{0} \quad\left(1.04 \pm 0.09 G_{0}+2.25 \pm\right.$ $0.20 G_{0} \rightarrow 3.32 \pm 0.30 G_{0}, \quad 2.25 \pm 0.20 G_{0}+2.25 \pm 0.20 G_{0} \rightarrow$ $4.44 \pm 0.25 G_{0}$, and a series combination of two $1.04 \pm$ $0.09 G_{0} \rightarrow 0.60 \pm 0.04 G_{0}$, and three $1.04 \pm 0.09 G_{0} \rightarrow 0.38 \pm$ $0.03 G_{0}$, via Eq. 2). Fig. $6 \mathrm{~b}$ shows the conductance histogram for CNT-B; a major peak occurs at $1.30 \pm 0.06 G_{0}$, and a very broad feature centred on $8.88 G_{0}$. The peak at $1.30 \pm 0.06 G_{0}$ coincides with one fundamental peak exhibited by all the MWCNT samples. Since one ideal SWCNT shell is predicted to support $2 G_{0}$ of conductance the very broad feature centred on $8.88 G_{0}$ indicates some nanotubes within the sample have $>4$ current-carrying walls. Fig. $6 \mathrm{c}$ shows the conductance histogram for CNT-C in terms of $G_{0}$. Resolved 
peaks were observed at $0.61 \pm 0.05 G_{0}, 1.28 \pm 0.08 G_{0}, 2.12 \pm$ $0.10 G_{0}, 3.14 \pm 0.26 G_{0}, 4.36 \pm 0.23 G_{0}$, and $6.31 \pm 0.09 G_{0}$. The low number of discrete peaks points to analysis via a simple parallel and series ballistic wire model for MWCNTs comprising a low number of current-carrying walls [20]. The simple model that emerges from this analysis is of two fundamental conductance values at $1.28 \pm 0.08 G_{0}$ and $2.12 \pm 0.10 G_{0}$, the higher value conductances are sums of combinations of the two fundamental peaks $\left(1.28 \pm 0.08 G_{0}+2.12 \pm 0.10 G_{0} \rightarrow 3.14 \pm 0.26 G_{0}, 2.12 \pm 0.10 G_{0}+\right.$ $2.12 \pm 0.10 G_{0} \rightarrow 4.36 \pm 0.23 G_{0}, 3.14 \pm 0.26 G_{0}+3.14 \pm 0.26 G_{0} \rightarrow$ $\left.6.31 \pm 0.09 G_{0}\right)$ and a series combination of two $1.28 \pm$ $0.08 \mathrm{G} 0 \rightarrow 0.61 \pm 0.05 \mathrm{G} 0$, again from Eq. 2 . Since the parallel combination of the two minor fundamental peaks is a major peak it can be concluded that the conduction pathway is within two walls of one nanotube (i.e. within 1 MWCNT). This simple model is very similar to that proposed for double-wall carbon nanotubes, namely: an outer-wall contribution of $\sim 1 G_{0}$ and a first inner-wall contribution of $2 G_{0}$ [20]. Fig. $6 d$ shows the conductance histogram for carboxylic acid functionalised MWCNTs (CNT-D); major peaks were observed at $0.56 \pm 0.08 G_{0}$, $0.87 \pm 0.10 G_{0}, 2.21 \pm 0.22 G_{0}$, and $3.28 \pm 0.08 G_{0}$. The conductance histogram for CNT-D shows fewer resolved peaks and a higher background conductance that extends to high conductance values. Peak values that coincide with those in Fig. 6a $\left(0.56 \pm 0.08 G_{0}, 1.35 \pm 0.07 G_{0}, 2.21 \pm 0.22 G_{0}\right.$, and $3.28 \pm 0.08 G_{0}$ ) can be described by the same model as above. However, the $0.87 \pm 0.10 G_{0}$ peak cannot be ascribed to a series or parallel combination of the other peaks so it must also be considered fundamental. Ballistic conductance testing of CNT-D and data interpretation has revealed the carboxyl-functionalised nanotubes are not electrically conductive due to the loss in the $\pi$-conjugated, electron de-localisation of the $\mathrm{sp}^{2} \mathrm{C}-\mathrm{C}$ bond in the nanotube walls, as a result of nanotube functionalization. In conclusion, the ballistic current pathways for CNT-C are restricted to the outer two layers of the MWCNT structure, the outer- and inner-layer supporting $\sim 1 G_{0}$ and $2 G_{0}$ of con- ductance, respectively. The CNT-D sample shows similar ballistic conductance characteristics with an additional $0.87 G_{0}$ contribution not seen in the other samples but the high background is evidence for non-ballistic transport suggesting a degree of disruption in the crystalline quality of the samples relative to the other samples. The arc-discharge produced tubes (CNT-A and CNT-B) comprise two distinct groups: those with ballistic current carrying pathways in a $\sim 1 G_{0}$ layer and a significant fraction with ballistic current distributed over $>4$ layers. These tubes are dominated by low-conductance non-ballistic transport with a minor contribution from two-layer ballistic conductance as exhibited by the other samples, suggesting a relatively high defect density in their structure.

As expected, the electrical percolation increased as a function of sample thickness. Fig. 7 shows how $\rho_{\mathrm{c}}$ increased from 0.2 to $0.5 \mathrm{wt} . \%$ when the film thickness was increased from 100 to $250 \mu \mathrm{m}$. This effect may be attributed to a more 2D arrangement of the MWCNTs when reducing the sample thickness for which lower amounts of MWCNT are required to form a conductive network in the plane as the conductivity is measured.

It is well understood during oscillatory melt rheology measurements that behavior at low frequencies is very responsive to the changing nano/micro-structure of filled polymer composites, such that the formation of percolated filler networks and associated 'pseudo-solid' like properties can be studied. The percolative state of the nanotubes can be detected by a sudden increase in storage modulus $\left(G^{\prime}\right)$ or complex viscosity $\left(\eta^{*}\right)$ as the behavior of the material becomes less dependent on frequency as a function of increased filler concentration. Evidence for the formation of a rheological percolated network for all composites prepared was investigated using oscillatory shear rheology, see Figs. 8-11. Fig. 8a and b show the variation in $G^{\prime}$ and $\eta$ with frequency as a function of CNT-D ( $f$-MWCNT) loading. At low frequencies both $G^{\prime}$ and $\eta^{*}$ increased significantly with increased CNT-D loading, the former by more than 2 orders of magnitude (9077\%) at $0.1 \mathrm{rad} / \mathrm{s}$ and the

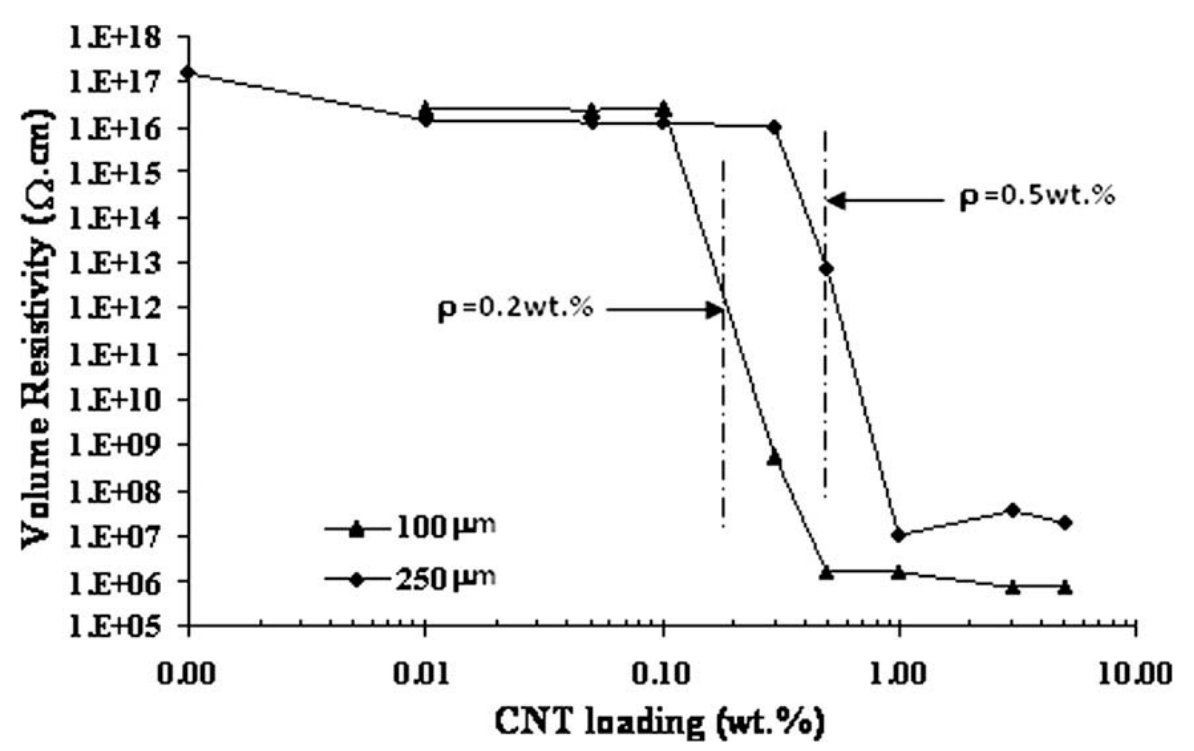

Fig. 7. Variation in volume resistivity as a function of CNT loading for different test sample thicknesses (100 and $250 \mu \mathrm{m})$ - PMMA/CNT-A composites. 

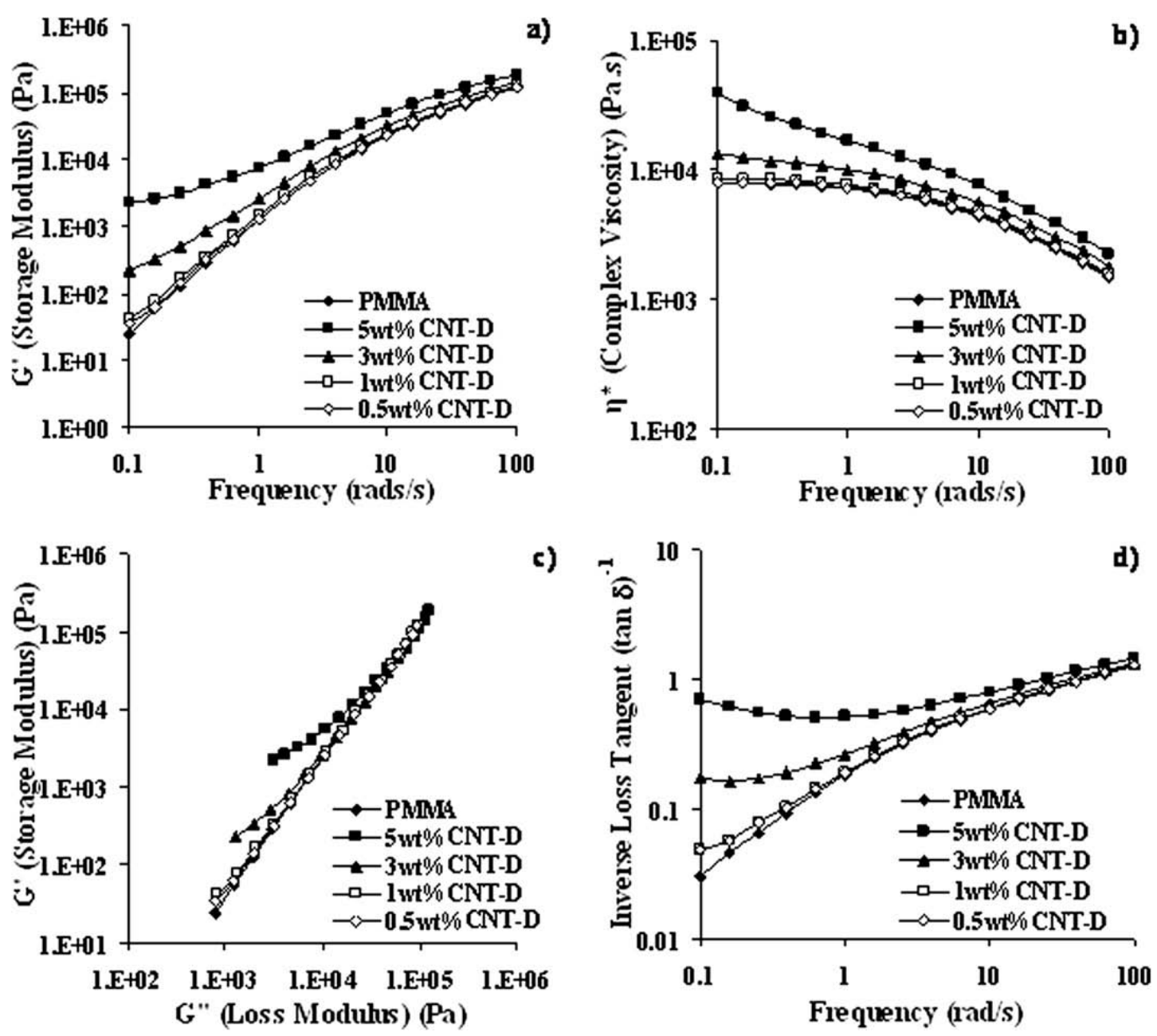

Fig. 8. Variation in (a) storage modulus $\left(G^{\prime}\right)$ as a function of frequency $(\omega)$, (b) complex viscosity $\left(\eta^{*}\right)$ as a function of $\omega$, (c) Cole-Cole plots $\left(G^{\prime}\right.$ versus $\left.G^{\prime \prime}\right)$ and $(\mathrm{d})$ inverse loss tangent $(\tan \delta)^{-1}$ as a function of $\omega$ for PMMA/CNT-D composites.

latter by $391 \%$ on addition of 5 wt.\% CNT-D, unambiguous evidence of the restriction of polymer flow by the formation of a CNT network in three dimensions throughout the composite. From Fig. 8b, the $5 \mathrm{wt} . \%$ composite exhibited a constant shear thinning effect during the entire frequency sweep, whereas the viscosity of the neat PMMA was independent of frequency at low values. The viscosity curve of the composites deviated from the behaviour of the neat polymer and became steeper at low frequencies. The increase in $\mathrm{G}^{\prime}$ and $\eta^{*}$ above nanotube loadings of $1 \mathrm{wt} . \%$ is indicative of the domination of polymer-nanotubepolymer interactions and the onset of formation of an interconnected structure of nanotubes. This is similar to that described by Pötschke et al. for a range of PC/MWCNT composites fabricated by a masterbatch dilution method having a rheological percolation at $2 \mathrm{wt}$ \% MWCNTs [39]. For the range of composites studied in this work, $G^{\prime}$ became less dependent on frequency as nanotube loading was increased above $1 \mathrm{wt}$ \% CNT-D, a phenomenon indicative of liquid-to-solid-like behaviour as the nanotube network restrains the long range motion of the polymer chains. The onset of percolation can be more readily identified by plotting $\log G^{\prime}$ versus $\log G^{\prime \prime}$ (Cole-Cole plot) and inverse $\tan \delta$ against frequency $(\omega)$, see Fig. 8c and $d$, respectively. Any deviation from a linear relationship between $G^{\prime}$ and $G^{\prime \prime}$ is indicative of the formation of a percolated network. This is clearly evident for the PMMA/CNT$\mathrm{D}$ composites as a percolation threshold was detected between 1 and 3 wt.\% CNT-D. Further evidence for network formation was apparent from a plot of inverse $\tan \delta$ versus frequency. An increase in $\tan \delta^{-1}$ is a measure of the increase in the 'solidity' of the composites [40]. It can be seen that at low frequencies and as CNT-D loading was increased from 1 to $3 \mathrm{wt} . \%$, and subsequently to $5 \mathrm{wt} . \%$, $\tan \delta^{-1}$ increased and both curves formed a plateau, suggesting percolation is closer to $3 \mathrm{wt} . \%$. This result is supported by our observations from exhaustive characterization of PMMA/CNT-D composites by HRTEM across the length scales; see Fig. 1e and f. As long as the inter-nanotube distance is equal to or greater than the average radius of gyration of the polymer chains, then chain mobility will be restricted. The average radius of gyration of the PMMA used in this study was estimated from:

$\left\langle S^{2}\right\rangle=a M^{b}$

where $\left\langle S^{2}\right\rangle$ is the mean squared radius of gyration, $M$ is molecular weight, in this instance $85,000 \mathrm{~g} / \mathrm{mol}$, and $a$ and $b$ are constants, 0.0713 and 1.0098 , respectively [41]. 

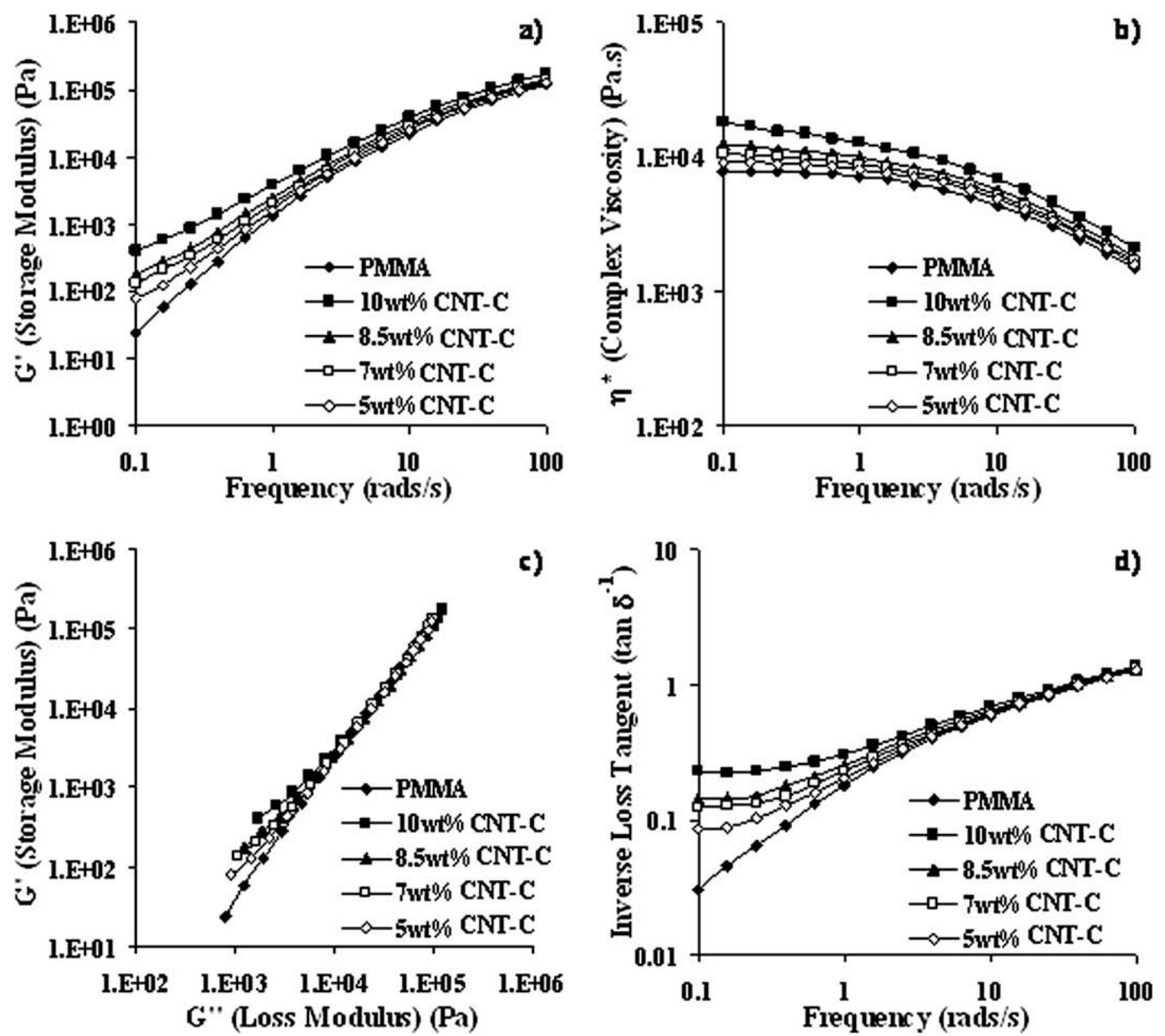

Fig. 9. Variation in (a) storage modulus $\left(G^{\prime}\right)$ as a function of $\omega$, (b) complex viscosity $\left(\eta^{*}\right)$ as a function of $\omega$, (c) Cole-Cole plots ( $G^{\prime}$ versus. $\left.G^{\prime \prime}\right)$ and (d) inverse loss tangent $(\tan \delta)^{-1}$ as a function of $\omega$, for PMMA/CNT-C composites.

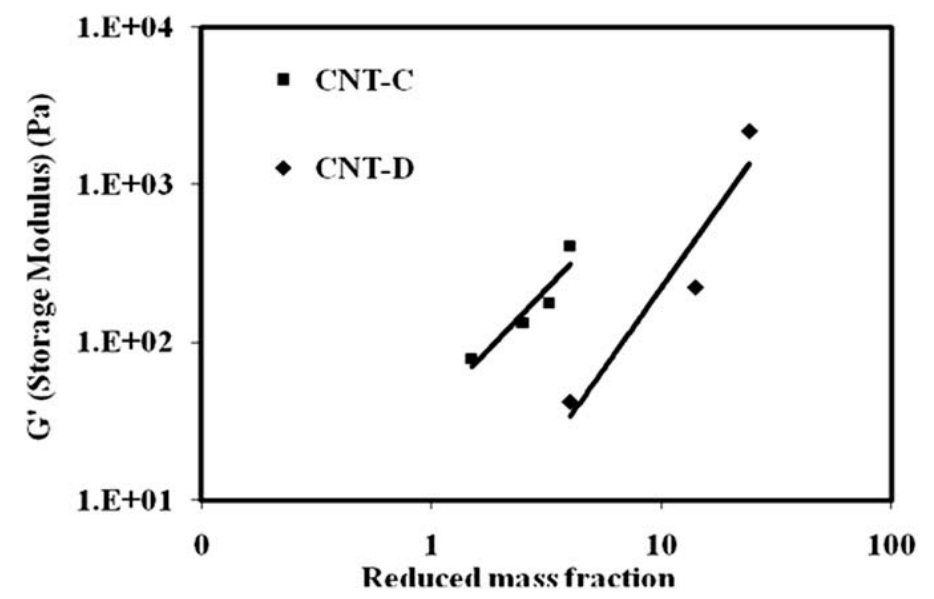

Fig. 10. Log-log plot of storage modulus $\left(G^{\prime}\right.$ at $\left.0.1 \mathrm{rad} / \mathrm{s}\right)$ versus reduced mass fraction for PMMA/CNT-C and PMMA/CNT-D composites.

The average diameter for a random coil was estimated to be $15.3 \mathrm{~nm}$. Achieving a percolation for CNT-D composites was in part surprising as CNT-D had the smallest average aspect ratio (100) of the 4 MWCNT types used, see
Table 1. This would indicate that interactions between the carboxylic acid groups on the CNT surface and PMMA are more critical to attaining rheological percolation rather than physical bridging of polymer chains. Such 

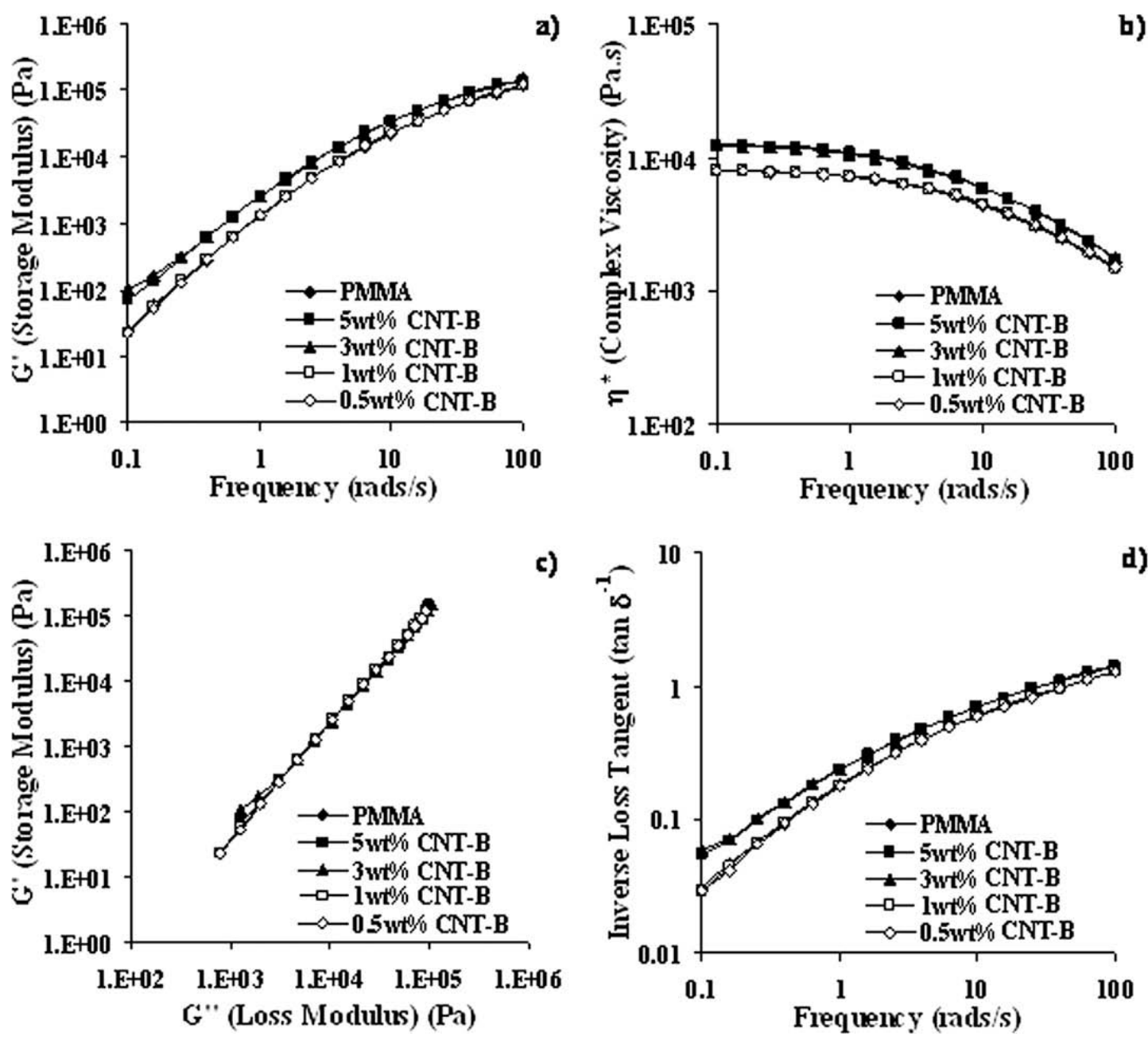

Fig. 11. Variation in (a) storage modulus $\left(G^{\prime}\right)$ as a function of $\omega$, (b) complex viscosity ( $\eta^{*}$ ) as a function of $\omega$, (c) Cole-Cole plots ( $G^{\prime}$ versus $\left.G^{\prime \prime}\right)$ and (d) inverse loss tangent $(\tan \delta)^{-1}$ as a function of $\omega$ for PMMA/CNT-B composites.

phenomena, i.e. rheological percolation but no electrical percolation, has been observed for polycarbonate MWCNT composites [42]. The role of reactive compatibilization and functionalized CNTs on electrical and rheological percolation has also been demonstrated for melt mixed polyamide-12 SWCNT systems [43]. If the average aspect ratio is much larger, as in the case for CNT-C (517), the restriction of the mobility of PMMA chains is still sufficient to form a rheological percolated network, see Fig. 9. The data presented in Fig. 9 for composites of PMMA (powder) with CNT-C shows the variation in $G^{\prime}, \eta^{*}$ and $\tan \delta^{-1}$ with frequency, and a plot of $\log G^{\prime}$ versus $\log G^{\prime \prime}$. Both $G^{\prime}$ and $\eta^{*}$ increased with successive addition of CNT-C up to $10 \mathrm{wt} . \%$, the former by $127 \%$ for $0.1 \mathrm{rad} / \mathrm{s}$. The definite onset of a rheological percolation was obvious from a $\log -\log$ plot of $G^{\prime}$ versus $G^{\prime \prime}$, (Cole-Cole plot), the change in slope and divergence of the line indicative of percolation behavior at low frequencies. Furthermore, a significant increase in $\tan \delta^{-1}$ was obtained confirming the formation of a percolated network at some loading approaching $5 \mathrm{wt} . \%$ CNT-C. A more accurate estimate of the rheological percolation for the composites of PMMA with CNT-C and CNT-D can be determined using a power law relationship:
$G^{\prime} \alpha\left(m-m_{c}\right)^{t}$

where $G^{\prime}$ is the storage modulus, $m$ is the MWCNT mass fraction, $m_{c}$ is percolation threshold and $t$ is the critical exponent which can be extracted from the slope of the line of a $\log -\log$ plot of $G^{\prime}$ versus reduced mass fraction $\left(m-m_{c}\right) / m_{c}$, see Fig. 10. Using this approach the rheological percolation threshold for PMMA/CNT-C and PMMA/CNT-D composites was found to be 1.52 and $2.06 \mathrm{wt} \%$, respectively.

Interestingly, for the composites prepared with CNT-C but with the PMMA in pellet form prior to melt mixing (not shown), $G^{\prime}$ was 1.5 times smaller and a rheological percolation threshold of $7 \mathrm{wt} . \%$ was obtained, in contrast to the composites prepared with PMMA in powder form. The former facilitates better dispersion and distribution of CNT-C in the matrix via network formation, whereas composites with a larger number of MWCNT agglomerations have melt-flow properties similar to neat PMMA. The effect of degree of dispersion on rheological properties of PMMA/SWCNT composites was reported by Du et al. [41]. The authors reported significantly higher $G^{\prime}$ values for a well dispersed PMMA/SWCNT (1 wt.\%) composite relative to a similarly poorly mixed composite. 

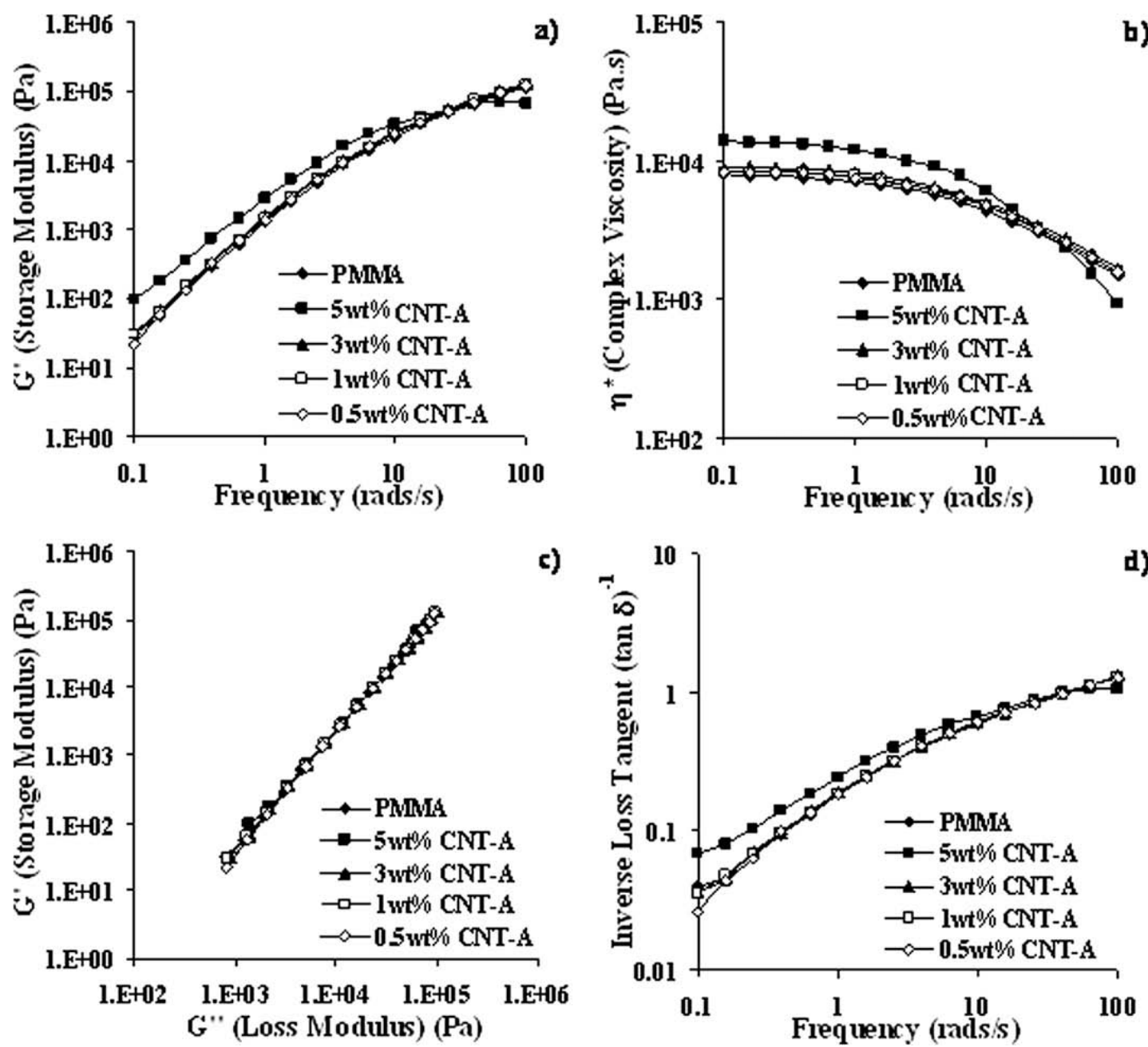

Fig. 12. Variation in (a) storage modulus $\left(G^{\prime}\right)$ as a function of $\omega$, (b) complex viscosity ( $\left.\eta^{*}\right)$ as a function of $\omega$, (c) Cole-Cole plots ( $G^{\prime}$ versus $\left.G^{\prime \prime}\right)$ and (d) inverse loss tangent $(\tan \delta)^{-1}$ as a function of $\omega$ for PMMA/CNT-A composites.

Evidence for the formation of a rheological percolated network for the composites prepared with AD MWCNTs having aspect ratios intermediate of CNT-C and CNT-D (i.e. CNT-A(300) and CNT-B(442)) was not conclusive for the MWCNT loadings studied. Fig. 11 shows $G^{\prime}, \eta^{*}$ and $\tan \delta^{-1}$ as a function of frequency $(\omega)$ and $\log G^{\prime}$ versus $\log G^{\prime \prime}$ for increasing CNT-B loading. A small increase in $G^{\prime}, \eta^{*}$, and $\tan \delta^{-1}$ across the frequency range studied was obtained for a CNT-B loading of $3 \mathrm{wt} . \%$ and above and a plot of $\log G^{\prime}$ versus $\log G^{\prime \prime}$ showed that the microstructure of the composite changed little on addition of CNT-B. Similar behavior was observed for the composites of PMMA with CNT-A, see Fig. 12. Minimal increases in $G^{\prime}, \eta^{*}$, and $\tan \delta^{-1}$ were observed, but in this instance only for a CNT-A loading approaching $5 \mathrm{wt} . \%$. The damping characteristics of the PMMA were not altered on addition of CNT-A. Although CNT-A and CNT-B have average aspect ratios intermediate of CNT-C and CNT-D, they also contain higher levels of impurities (including various carbonaceous materials), $85 \%$ and $60 \%$, respectively, see Table 1 . The PMMA/CNT-A composites formed an electrical percolated network as the CNT-A tubes were highly dispersed in the PMMA matrix but did not form a rheological percolated network.
The more pure CNT-B tubes were poorly mixed in the PMMA matrix (Fig. 1d) and didn't form either an electrical or rheological percolated network. The CNT-B tubes were coated with an insulating PMMA layer, which grossly affected the electron conduction process.

\section{Conclusions}

Composites of PMMA with four different MWCNTs of varying aspect ratio and functionality were prepared using melt mixing in a twin-screw extruder. For a given MWCNT, the initial particle size of the PMMA used was found to be critical as to whether an electrical or rheological percolated network was formed. By way of example, when CNT-C was added at loadings up to $10 \mathrm{wt}$.\% to PMMA (particle diameter $\sim 2 \mathrm{~mm}$ ) no electrical percolation was obtained. In contrast, an electrical percolation threshold of $7.75 \mathrm{wt} . \%$ was attained when the average PMMA particle size was $250 \mu \mathrm{m}$ and the electrical resistivity of PMMA decreased by about 11 orders of magnitude from $10^{17}$ to $10^{6} \mathrm{Ohm}$ $\mathrm{cm}$. The smaller particle size facilitates more intimate mixing of polymer chains and nanotubes under the conditions 
of melt mixing used in the extruder. Both set of composites formed rheological percolated networks characteristic of pseudo-solid-like behaviour but with different thresholds, the former at about $4 \mathrm{wt} . \%$ and the latter at approximately 7 wt.\%. The lowest electrical percolation threshold (0.5 wt.\%) was detected for the composites prepared with CNT-A. Presumably the short and rigid arc-discharge produced CNT-A tubes are more easily dispersed and distributed in the PMMA matrix. As the CNT-A sample was 15\% pure and assuming only the metallic tubes contributed to the formation of an electrical network, the threshold for this system maybe as low as $0.075 \mathrm{wt} . \%$, significantly below that previously reported for other polymer-MWCNT melt mixed systems. Addition of CNT-B or CNT-D to PMMA up to $10 \mathrm{wt} . \%$ did not alter the electrical resistivity of PMMA.

Interestingly, the lowest aspect ratio and shortest tubes (CNT-D) readily formed a rheological percolated network in PMMA, indicating interfacial interactions between PMMA chains and the carboxylic acid functional groups on the surface of CNT-D play a major role in the formation of a rheological percolated microstructure. The rheological percolation threshold for the composites with CNT-C and CNT-D, determined using a power law relationship, were 1.52 and $2.06 \mathrm{wt} . \%$, respectively.

The formation of a rheological percolated network and not an electrical equivalent for the PMMA/CNT-D composites was a consequence of the loss of $\pi$-conjugation and electron de-localisation of the $\mathrm{sp}^{2} \mathrm{C}-\mathrm{C}$ bonds from carboxylic acid functionalization of pristine CNT-D tubes. Functionalization destroys the crystalline quality of CNT-D resulting in non-ballistic transport in these tubes. In addition, the strong interactions towards the PMMA may form an insulating polymer layer around the nanotubes, so that a rheological percolation indicates network formation but this network is not electrically conductive. The formation of rheological percolated networks for the MWCNTs with aspect ratios intermediate of CNT-D (smallest) and CNT-C (largest) was not obvious for the composition range studied. Sufficiently long and/or functionalized tubes are required to physically bridge or interact with PMMA to alter polymer chain dynamics.

\section{Acknowledgement}

This work was supported by the EU Specific Targeted Research Project DESYGN-IT (No.NMP4-CT-2004-505626), The Royal Society (574006/G503/24135) and The Nuffield Foundation (NAL/00696/G).

\section{References}

[1] Jin Z, Pramoda KP, Xu G, Goh SH. Chem Phys Lett 2001;337:43-7.

[2] Thostenson ET, Chou T-W. J Phys D: Appl Phys 2002;35:S77-80.

[3] McNally T, Pötschke P, Halley P, Murphy M, Martin D, Bell SEJ, et al. Polymer 2005;46:8222-32.

[4] Pötschke P, Bhattacharyya AR, Janke AE. Polym J 2001;39:137-48.
[5] Manchado MAL, Valentini L, Biagiotti J, Kenny JM. Carbon 2005:43:1499-505.

[6] Wanjale SD, Jog JP. Polymer 2006;47:6414-21.

[7] Gorga RE, Cohen RE. J Polym Sci Part B: Polym Phys 2004;42: 2690-702.

[8] Haggenmueller R, Gommans HH, Rinzler AG, Fischer JE, Winey KI. Chem Phys Lett 2000;330:219-25.

[9] Zhou Z, Wang S, Lu L, Zhang Y, Zhang Y. Comp Sci Tech 2007;67:1861-9.

[10] Jin Z, Pramoda KP, Goh SH, Xu G. Mater Res Bull 2002;37:271-8.

[11] Park SJ, Kim ST, Cho MS, Kim HM, Joo J, Choi HJ. Curr Appl Phys 2005;5:302-4.

[12] Kim HM, Kim K, Lee SJ, Joo J, Yoon HS, Cho SJ, et al. Curr Appl Phys 2004;4:577-80.

[13] Benoit, JM, Corraze B, Lefrant S, Blau WJ, Bernier P, Chauvet O. Synth Met 2001;121:1215-6.

[14] Du F, Fischer JE, Winey KI. J Polym Sci Part B: Polym Phys 2003;41:3333-8.

[15] Sandler J, Shaffer MSP, Prasse T, Bauhofer W, Schulte K, Windle AH. Polymer 1999;40:5967-71

[16] Sandler JKW, Kirk JE, Kinloch IA, Shaffer MSP, Windle AH. Polymer 2003;43:5893-9.

[17] Martin CA, Sandler JKW, Shaffer MSP, Schwarz M-K, Bauhofer W, Schulte K, et al. Comp Sci Technol 2004;64:2309-16.

[18] Li C, Thostenson ET, Chou TW. Appl Phys Lett 2007;91:22311401-403.

[19] Bravo VL, Hrymak AN, Wright JD. Polym Eng Sci 2000;40:525-41.

[20] Baxendale M, Melli M, Alemipour Z, Pollini I, Dennis TJS. J Appl Phys 2007;102:10372101-106.

[21] Poncharal P, Berger C, Yi Y, Wang ZL, de Heer WA. J Phys Chem B 2002;106:12104-8.

[22] Frank S, Poncharal P, Wang ZL, de Heer WA. Science 1998;280:1744-6.

[23] Urbina A, Escheverria I, Perez-Garrido A, Diaz-Sanchez A, Abellan J. Phys Rev Lett 2003;90:10660301-304.

[24] Li HJ, Lu WG, Li JJ, Bai XD, Gu CZ. Phys Rev Lett 2005;95:08660101-104.

[25] Bourlon B, Glatti DC, Plaçais B, Berroir JM, Miko C, Forrò L, et al. Phys Rev Lett 2004;92:02680401-404.

[26] Tsutsui M, Mitsuya S, Kurosawa S, Sakai A. Nanotechnology 2005; $16: 1863-7$

[27] (a) de Heer WA, Frank S, Ugarte DZ. Phys Rev B 1997;104:469-473; (b) Berger, C, Yi Y, Gezo J, Poncharal P, de Heer WA. New J Phys 2003;5:158.1-158.16; (c) de Heer WA, Ugarte D. in: Nanowires, editors, Kluwer, Netherlands, 1997, p 227.

[28] Büttiker M. IBM J Res Dev 1988;32:63.

[29] Landauer, R. IBM J Res Dev 1957;1:223.

[30] Landauer R. Philos Mag 1970;21:863.

[31] Saito R, Dresselhaus G, Dresselhaus MS. Physical properties of carbon nanotubes. Imperial College Press: London; 1998 (Chapter 4).

[32] Chico L, Benedict LX, Louie SG, Cohen ML. Phys Rev B 1996;54:2600-6.

[33] Gojny FH, Nastalczyk J, Rslaniec Z, Schulte K. Chem Phys Lett 2003;370:820-4.

[34] Ramanathan T, Liu H, Brinson LC. J Polym Sci Part B: Polym Phys 2005;43:2269-79.

[35] Zhou O, Fleming RM, Murphy DW, Chen CH, Haddon RC, Ramirez AP, Glarum SH. Science 1994;263:1744-7.

[36] Dresselhaus MS, Dresselhaus G, Sugihara K, Spain IL, Goldberg H. Graphite fibres and filaments. Springer-Verlag: NewYork; 1988.

[37] Valentini L, Biagiotti J, Kenny JM, Santucci S. J Appl Polym Sci 2003;87:708-13.

[38] Gojny FH, Wichmann MHG, Fiedler B, Kinloch IA, Bauhofer W, Windle AH, et al. Polymer 2006;47:2036-45.

[39] Pötschke P, Fornes TD, Paul DR. Polymer 2002;43:3247-55.

[40] Kota AK, Cipriano BH, Duesterberg MK. Gershon AL, Powell D, Raghavan SR, et al. Macromolecules 2007;40:7400-7406.

[41] Du F, Scogna RC, Zhou W, Brand S, Fischer JE, Winey KI. Macromolecules 2004;37:9048-55.

[42] Pötschke P, Abdel-Goad M, Alig A, Dudkin S, Lellinger D. Polymer 2004;45:8863-70.

[43] Bhattacharyya AR, Pötschke P, Abdel-Goad M, Fischer D. Chem Phys Lett 2004;392:28-33. 